\title{
Reduced serum methods for contact-based coculture of human dermal fibroblasts and epidermal keratinocytes
}

\author{
Snehal Kadam1 iD, Madhusoodhanan Vandana1 iD \& Karishma S Kaushik*,1 iD \\ ${ }^{1}$ Institute of Bioinformatics \& Biotechnology, Savitribai Phule Pune University, India; *Author for correspondence: karishmaskaushik@gmail.com
}

BioTechniques 69: 00-00 (November 2020) 10.2144/btn-2020-0112

First draft submitted: 20 July 2020; Accepted for publication: 10 August 2020; Published online: 1 September 2020

\section{ABSTRACT}

Direct contact-based coculture of human dermal fibroblasts and epidermal keratinocytes has been a long-standing and challenging issue owing to different serum and growth factor requirements of the two cell types. Existing protocols employ high serum concentrations (up to $10 \%$ fetal bovine serum), complex feeder systems and a range of supplemental factors. These approaches are technically demanding and labor intensive, and pose scientific and ethical limitations associated with the high concentrations of animal serum. On the other hand, serum-free conditions often fail to support the proliferation of one or both cell types when they are cultured together. We have developed two reduced serum approaches (1-2\% serum) that support the contact-based coculture of human dermal fibroblasts and immortalized keratinocytes and enable the study of cell migration and wound closure.

\section{LAY ABSTRACT}

Two reduced serum-based methods (1-2\% fetal bovine serum) were formulated using commercially available media components. Human dermal fibroblasts and epidermal keratinocytes were cocultured by layering keratinocytes over confluent fibroblasts or by seeding the two cell types simultaneously. The coculture platform was leveraged to study cell migration and wound closure using scratch assays.

\section{TWEETABLE ABSTRACT}

To serum or not to serum? Two new reduced serum approaches for study of cell migration and wound closure.

\section{METHOD SUMMARY}

Two reduced serum-based methods (1-2\% fetal bovine serum) were formulated using commercially available media components. Human dermal fibroblasts and epidermal keratinocytes were cocultured by layering keratinocytes over confluent fibroblasts or by seeding the two cell types simultaneously. The coculture platform was leveraged to study cell migration and wound closure using scratch assays.

\section{GRAPHICAL ABSTRACT}
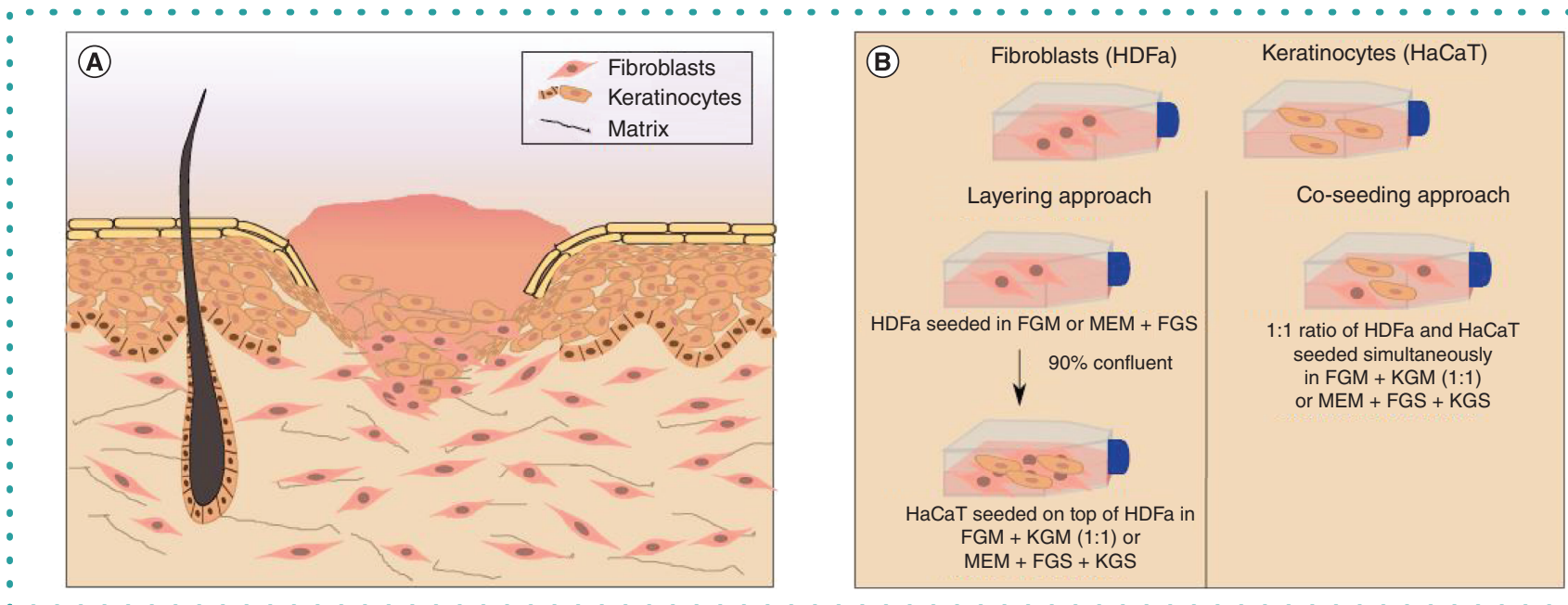

\section{KEYWORDS:}

coculture $\bullet$ fetal bovine serum $\bullet$ fibroblasts $\bullet$ keratinocytes $\bullet$ reduced serum $\bullet$ wound bed 


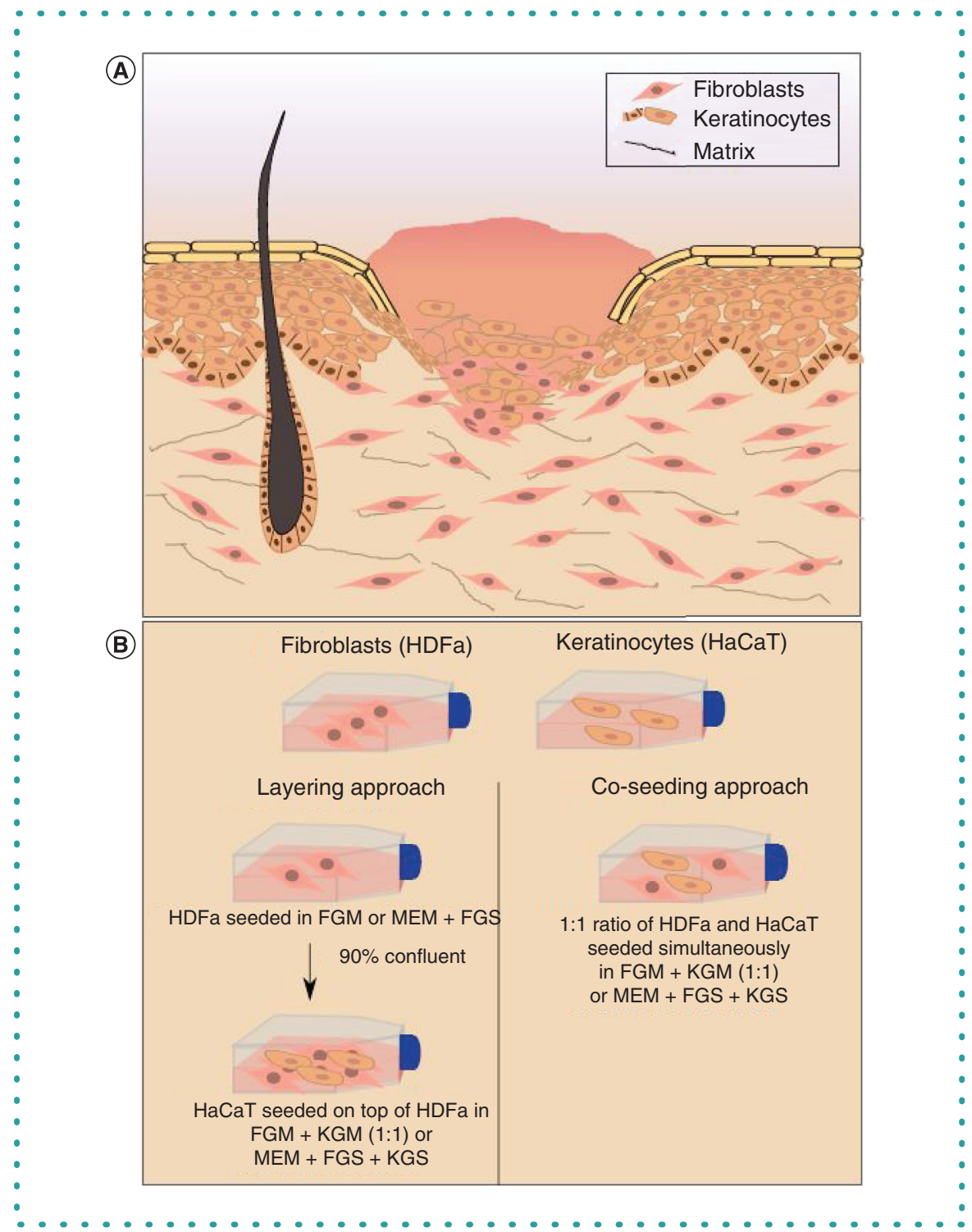

Figure 1. Contact-based coculture of fibroblasts and keratinocytes is important to study the wound bed. (A) In the proliferative stage of wound healing, the wound bed consists of fibroblasts and keratinocytes, in close association with each other. Fibroblasts from the wound edges migrate into the wound bed, establishing signaling networks that recruit adjacent keratinocytes. Keratinocytes migrate from the wound edge and from around epithelial appendages, resulting in distinct regions of fibroblast-keratinocyte interactions. (B) Schematic of two reduced serum conditions developed to coculture fibroblasts (HDFa) and keratinocytes (HaCaT), based on a mixture of specialized cell media (FGM+KGM) or minimal media supplemented with cell-specific growth factors supplements (MEM+FGS+KGS), using layering and co-seeding approaches. FGM: Fibroblast growth medium; FGS: Fibroblast growth supplement; KGM: Keratinocyte serum-free growth medium; KGS: Keratinocyte growth supplement; MEM: Minimal essential medium.

In the proliferative phase of wound healing, the wound bed is progressively filled with granulation tissue [1], followed by re-epithelialization to restore surface integrity [2]. Granulation tissue consists of, among other components, fibroblasts from the dermis, which proliferate and migrate into the wound bed [3]. Fibroblasts secrete signaling factors that recruit adjacent epidermal keratinocytes from the wound edge and epithelial appendages (Figure 1A) $[3,4]$. Migration of keratinocytes and fibroblasts results in regions of direct contact between the two cell types [5,6], observed as both cells coexisting in the wound bed or as keratinocytes lying on top of fibroblasts, as seen at the wound edges. Cell-to-cell interactions between these two cell types critically impact cellular proliferation, differentiation and migration and thereby affect wound bed structure and functions [6-9].

Contact-based coculture of human dermal fibroblasts (fibroblasts) and epidermal keratinocytes (keratinocytes) has been a challenging issue in the field of wound and skin studies (Table 1) [10-19]. Fibroblasts and keratinocytes require a host of growth factors and sup- 


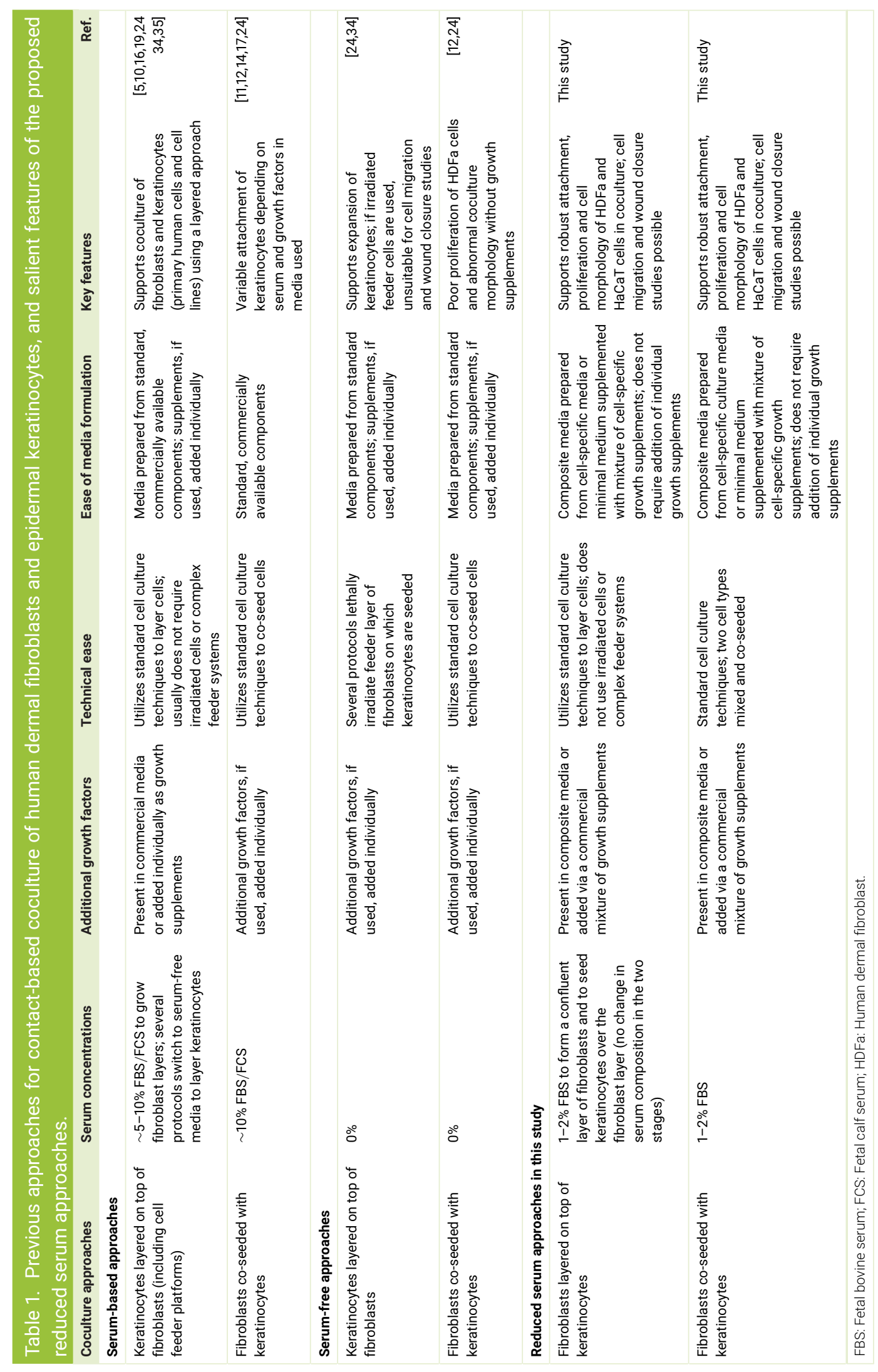


plements to support their in vitro proliferation [20-23], the concentrations and compositions of which vary between the two cell types. Animal serum (fetal bovine serum [FBS] or fetal calf serum) is an undefined mixture of various growth supplements widely used in cell culture media [10,12-14,17,24]; however, given the scientific, technical and ethical limitations associated with its use, there is a global push to reduce the usage of animal serum in cell culture studies. Further, serum poses a special concern in the context of fibroblast and keratinocyte coculture. The presence of serum is required to support the proliferation of fibroblasts, in contrast to primary keratinocytes, which typically grow poorly or fail to attach and grow in the presence of serum and are therefore cultivated in serum-free media [16,25-30]. Several wound and skin studies have overcome this problem by using immortalized human keratinocyte cell lines, such as HaCaT cells [29,31], which are amenable to long-term passage and display better serum tolerance, as a model for keratinocyte functions [30,32,33]. While HaCaT cells are more tolerant to serum than primary human keratinocytes, they have been shown to demonstrate impaired proliferation in coculture with fibroblasts at high concentrations of serum, such as $10 \%$ FBS, which is the concentration usually employed for fibroblast growth [12].

Taken together, existing approaches for contact-based coculture of fibroblasts and keratinocytes are not only technically demanding and labor intensive, but are also limited by the differential effects of serum on the two cell types, the need for complex cell feeder platforms, and material-intensive media formulations (Table 1) [24,34-38].

\section{Materials \& methods}

\section{Cell culture \& maintenance}

Adult primary human dermal fibroblasts (HDFa) were obtained from PromoCell (Germany) and maintained in all-in-one, ready-to-use Fibroblast Growth Media (FGM) (Cell Applications 116-500) containing 2\% FBS. Immortalized adult human keratinocytes (HaCaT) were a gift from Madhur Motwani (Linq Labs, Pune, India) and were maintained in an all-in-one, ready-to-use Keratinocyte Serum-free Growth Medium (KGM) (Cell Applications 131-500A). Cells were maintained at $37^{\circ} \mathrm{C}$ in a $5 \% \mathrm{CO}_{2}$ humidified incubator. Coculture was done in T25 flasks (Tarsons) and six-well tissue culture-treated plates (Thermo Scientific). Cell counting was done with a hemocytometer. Imaging was performed at magnification $100 \times$ with a Magnus INVI microscope equipped with a Magcam DC5 camera. For all experiments in minimal essential medium (MEM), penicillin and streptomycin (Gibco) was added to media in a final concentration of 1×.

\section{Reduced serum media formulations for coculture of HDFa \& HaCaT cells \\ Reduced serum formulations based on all-in-one fibroblast \& keratinocyte growth media}

Equal parts of FGM (containing $2 \%$ serum) and KGM were mixed, resulting in a composite medium with an effective concentration of $1 \%$ serum (referred to as FGM+KGM in this study). FGM and KGM are commercially available formulations, serving as an 'all-inone' composition of growth factors, supplements and antibiotics. Notably, FGM contains $2 \%$ FBS, whereas KGM is serum-free. Media formulations were freshly prepared prior to each use.

\section{Reduced serum formulations based on MEM with cell-specific growth supplements}

MEM containing Earle's salts, $2 \mathrm{mM}$ L-glutamine, $1 \mathrm{mM}$ sodium pyruvate, nonessential amino acids and $1.5 \mathrm{~g} / \mathrm{l}$ sodium bicarbonate (Himedia, AL047S), was supplemented with Low Serum Growth Supplement for Fibroblasts (Gibco, S00310, referred to as Fibroblast Growth Supplement [FGS] in this study) and Human Keratinocyte Growth Supplement (Gibco, S0015, referred to as Keratinocyte Growth Supplement [KGS] in this study) at concentrations of $1 \times$ each; the resultant medium is referred to here as MEM+FGS+KGS. FGS contains fetal bovine serum, hydrocortisone, human EGF, basic FGF and heparin. When FGS is added to the medium at a concentration of $1 \times$, the resultant medium contains 2\% FBS (information from manufacturer). KGS contains bovine pituitary extract, recombinant human IGF-1, hydrocortisone, bovine transferrin and human EGF, and is serum-free. Media formulations were freshly prepared prior to each use.

\section{Coculture of HDFa \& HaCaT cells with layering \& co-seeding approaches}

For the layering approach, HDFa cells were counted ( $\sim 10^{5}$ cells) and seeded in tissue culture-treated T25 flasks in FGM or MEM supplemented with $1 \times \mathrm{FGS}$ (both containing $2 \%$ serum), incubated at $37^{\circ} \mathrm{C}$ in a $5 \% \mathrm{CO}_{2}$ incubator and grown to $90 \%$ confluency. When the cells were confluent, the medium was removed and replaced with FGM+KGM (in a 1:1 ratio; 1\% serum) or MEM containing both FGS and KGS (MEM+FGS+KGS, $1 \times$ each, $2 \%$ serum). HaCaT cells were counted ( $\sim 10^{5}$ cells) and seeded on the confluent layer of fibroblasts. Flasks were imaged with a $10 \times$ objective (total magnification $100 \times$ ) every $24 \mathrm{~h}$ for 5 days.

For the co-seeding approach, HDFa and HaCaT cells were counted and $\sim 10^{5}$ cells of each type were mixed (resulting in a 1:1 ratio) in $\mathrm{FGM}+\mathrm{KGM}$ ( $1 \%$ serum) or MEM+FGS+KGS ( $2 \%$ serum), and co-seeded into $\mathrm{T} 25$ flasks. Co-seeded cells were incubated at $37^{\circ} \mathrm{C}$ in a $5 \% \mathrm{CO}_{2}$ incubator. The T25 flasks were imaged with a $10 \times$ objective (total magnification $100 \times$ ) every $24 \mathrm{~h}$ for 5 days.

\section{Wound scratch assay on cocultured platforms}

HDFa and HaCaT were seeded in six-well tissue culture-treated plates under reduced serum conditions using the co-seeding or layering approaches. When the cocultured cells reached $90 \%$ confluency, the medium was replaced with fresh reduced serum medium (FGM+KGM or MEM+FGS+KGS), and a scratch was made across each well using a sterile 200- $\mu$ l pipette tip. Following this, the wells were imaged with a $10 \times$ objective (total magnification $100 \times$ ) at $0,4,24$ and $48 \mathrm{~h}$ (three images were captured per scratch at different 
regions). The images were analyzed in ImageJ [39] by drawing a freehand outline of the scratch and using ImageJ tools to calculate the area within the outlined region. This was done for three images per well for three replicates for each condition, and an average wound area was calculated. Percentage of wound closure percentage was calculated as

$$
\% \text { wound closure }=\left(\frac{\left(\mathrm{W}_{0}-\mathrm{W}_{\mathrm{x}}\right)}{\mathrm{W}_{0}}\right) \times 100
$$

where $W_{0}=$ wound area at $0 \mathrm{~h}$ and $\mathrm{W}_{\mathrm{x}}=$ wound area at ' $\mathrm{x}$ ' $\mathrm{h}$ (calculated at $0,4,24$ and $48 \mathrm{~h}$ ).

\section{Results \& discussion}

Reduced serum conditions developed using commercial media components

To coculture HDFa and $\mathrm{HaCaT}$ cells, two reduced serum media were formulated using commercially available media components (Figure 1B). These formulations result in effective concentrations of $1 \%$ FBS in FGM+KGM and $2 \%$ FBS in MEM+FGS+KGS, representing a five- to tenfold reduction in serum concentration as compared with previous studies (that use 5-10\% serum) [10-12,14-16,19,40]. In addition to reduced serum concentrations, our formulations also contain combinations of cell-specific growth factors and supplements, recommended for fibroblast and keratinocyte culture. These reduced serum formulations are simple to develop and use widely available media components. Notably, we have leveraged commercial 'all-in-one' formulations (of media and growth supplements) to develop reduced serum concentrations and incorporate cell-specific growth supplements. This is in contrast to previous approaches for fibroblast and keratinocyte coculture, which add serum (most often 10\%), growth factors and supplements individually to the cell culture media; this approach is significantly more resource, time, and labor intensive $[10,11,15,16,19,24]$. Although the KGS is serum-free, it does contain bovine pituitary extract $(0.2 \% \mathrm{v} / \mathrm{v}$, an undefined animal product); however, the resultant formulation (MEM+FGS+KGS) contains very low concentrations of this product.

\section{Reduced serum conditions support the coculture of HDFa \& HaCaT cells using the layering technique}

For the layering approach, HDFa cells were seeded and grown to $90 \%$ confluency in tissue culture-treated T25 flasks, following which $\mathrm{HaCaT}$ cells were counted and seeded on layers of confluent HDFa. HDFa cells were initially cultured in FGM (containing $2 \%$ FBS) or $\mathrm{MEM}+1 \times \mathrm{FGS}$ (final concentration $2 \%$ FBS), where they were observed to attach and proliferate, and formed confluent layers in 2-3 days under reduced serum conditions (Figure 2A). This is notable, given that previous protocols have employed media containing $10 \%$ FBS to grow these HDFa layers $[10,11,15,16,19]$ and HDFa cells show poor proliferation under serum-free conditions [12]. After HDFa layers grew to confluency, HaCaT cells were introduced in FGM+KGM (1\% FBS) and MEM+FGS+KGS (2\% FBS). Starting from day 1, HaCaT cells were observed to attach onto HDFa layers under both reduced serum conditions. As seen in Figure 2A, HaCaT cells formed distinct clusters on and between sheaths of HDFa cells from day 1 (MEM+FGS+KGS) or day 2 (FGM+KGM), with no observable difference in the coculture morphology between the two medium formulations. Our formulations enable layered coculture of HDFa and HaCaT cells using reduced serum concentrations at all stages of the protocol, and thereby represent an advancement to previous protocols that employ high-serum (10\% FBS or fetal calf serum) media and different media compositions (10\% FBS and subsequently serum-free) for the two cell types $[10,11,15,16,19]$.

\section{Reduced serum conditions support the coculture of HDFa \& HaCaT cells using the co-seeding technique}

Because the simultaneous attachment and proliferation of the two cell types is important to explore the range of cellular interactions in the wound bed $[4,11,12,17]$, we proceeded to employ a co-seeding approach in which both cell types were seeded simultaneously. HDFa and $\mathrm{HaCaT}$ were seeded in a 1:1 ratio in tissue culture-treated T25 flasks. Co-seeding was done in FGM+KGM (1\% serum) and in MEM+FGS+KGS (2\% serum). After $24 \mathrm{~h}, \mathrm{HDFa}$ and HaCaT were seen to attach and proliferate, and showed characteristic cell morphology which was similar across both reduced serum conditions (Figure 2B). Starting from day 2, the HaCaT cells were observed to cluster in colonies which became progressively dense and tightly packed, surrounded by sheaths of HDFa cells. The coculture platform became fully confluent by day 5 (Figure 2B).

In another study [10], human dermal fibroblasts and epidermal keratinocytes were cocultured in a layered fashion. In this approach, fibroblasts were cultured on six-well Falcon plates or cell culture inserts for $48 \mathrm{~h}$, after which keratinocytes were seeded on top of them. For the cultivation of fibroblasts, Roswell Park Memorial Institute 1640 medium containing 10\% FBS was used; when keratinocytes were added, the medium was switched to serum-free conditions (KGM with growth supplements). Our study represents a significant advance to this work, as we have successfully managed to coculture fibroblasts and keratinocytes with the layering approach using 1-2\% FBS at all stages of the protocol, including in the initial step of fibroblast culture. Further, our reduced serum approaches successfully cocultured the two cell types simultaneously using the co-seeding approach, which cannot be achieved with protocols that require two different media formulations.

A previous study reported attempts at layering and co-seeding approaches for HDFa and HaCaT coculture under serum-free conditions, using Dulbecco's modified Eagle medium with glutamine and antibiotics [12]. To achieve coculture, HaCaT cells were added soon after the introduction of HDFa cells ( $40 \mathrm{~min}$ ) or across 5 days. However, HDFa cells (seeded at a density of $5 \times 10^{3}$ cells $/ \mathrm{cm}^{2}$ ) showed 


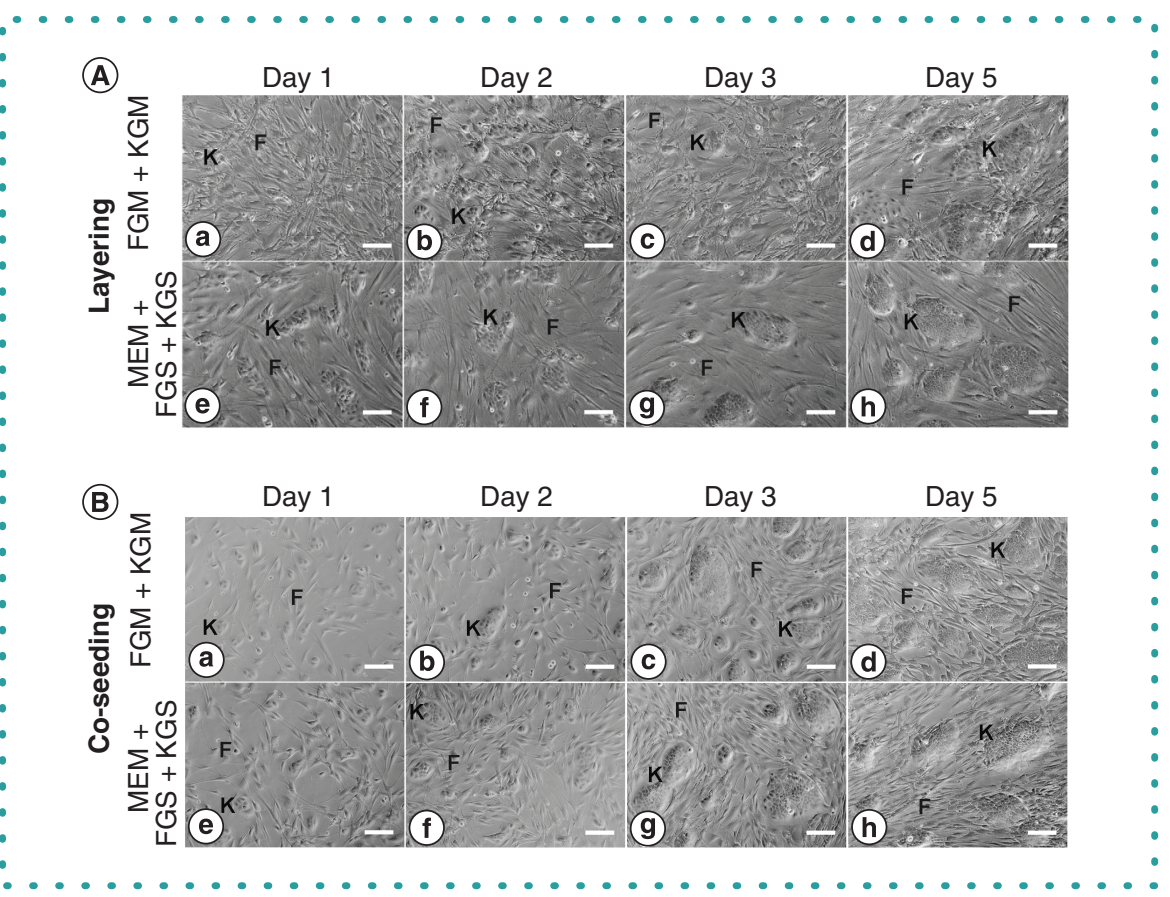

Figure 2. Coculture of fibroblasts and HaCaT cells (keratinocytes) using layering and co-seeding techniques under reduced serum (1-2\%) conditions. The two cell types were added in a 1:1 ratio. (A) When layered over confluent sheaths of HDFa, HaCaT cells form distinct clusters on HDFa cells starting from day 1 (MEM+FGS+KGS) or day 2 (FGM+KGM), which continue to proliferate and increase in size. (B) When co-seeded under both reduced serum conditions, $\mathrm{HaCaT}$ cells form distinct colonies by day 2 ; these become more dense and tightly packed over subsequent days, surrounded by sheaths of proliferating fibroblasts.

Scale bars $=150 \mu \mathrm{m}$.

FGM: Fibroblast growth medium; FGS: Fibroblast growth supplement; HDFa: Human dermal fibroblast; KGM: Keratinocyte serum-free growth medium; KGS: Keratinocyte growth supplement; MEM: Minimal essential medium.

significantly impaired proliferation under serum-free conditions in monoculture and coculture, even after 5 days of culture; even when the seeding density was increased, HDFa cells in coculture displayed impaired proliferation and failed to achieve confluency. For example, at the seeding density of $5 \times 10^{3}$ cells $/ \mathrm{cm}^{2}$, HDFa cells achieved a maximum confluency of $1.35 \%$ (vs $60 \%$ confluency under $10 \%$ FBS conditions). Even with increased seeding density $(80,000$ cells/ml), the maximum confluence achieved by HDFa cells was $44.87 \%$, and this density also supported HaCaT proliferation poorly in coculture. Furthermore, HaCaT cells also did not show the typical morphology and proliferation characteristics under these serum-free conditions. When quantified, at a seeding density of $5 \times 10^{3} \mathrm{cells} / \mathrm{cm}^{2}$, the maximum confluence achieved by $\mathrm{HaCaT}$ cells was $0.13 \%$; while proliferation of $\mathrm{HaCaT}$ cells improved at higher densities, the proliferation and confluence of HDFa remained suboptimal.

Our reduced serum approaches used seeding densities of $\sim 10^{5}$ cells, in a 1:1 ratio of the two cell types, and demonstrated successful coculture of HDFa and $\mathrm{HaCaT}$ cells. In the layering approach, HDFa cells grew to confluence in the reduced serum conditions in 2-3 days, and $\mathrm{HaCaT}$ cells added subsequently proceeded to form distinct clusters on and between sheaths of HDFa cells (Figure 2). With the co-seeding approaches, both cell types were seen to attach and proliferate, and the cocultured platform reached confluence in 5 days (Figure 2).

\section{Reduced serum conditions support characteristic HDFa \& HaCaT morphologies under coculture conditions}

In coculture under reduced serum conditions, HaCaT cells displayed typical polygonal cell clusters (colonies) with mosaic, cobblestone cell morphology and were surrounded by characteristic sheaths of spindle-shaped HDFa cells (Figure 2 \& Supplementary Figure 1A). This characteristic coculture arrangement was observed with both layering and co-seeding approaches and is similar to that seen in previous coculture studies with $10 \%$ FBS or serum-free conditions with a range of growth supplements $[12,17,24]$. Serial images from days 1-5 (Figure 2) show both cell types displaying robust attachment and subsequent proliferation (reaching confluency in the coseeding approach) in coculture under reduced serum conditions. This is seen as progressively enlarging clusters of HaCaT cells and the formation of HDFa sheaths around HaCaT colonies.

It is important to note that the reduced serum conditions in this study include cell-specific growth supplements (either as components of the 'all-in-one' specialized cell media or as a mixture of growth supplements added to minimal media) which, along with optimum serum concentrations, were observed to be critical to establish coculture. As seen in Supplementary Figure 1B, when co-seeded in the 
(A)

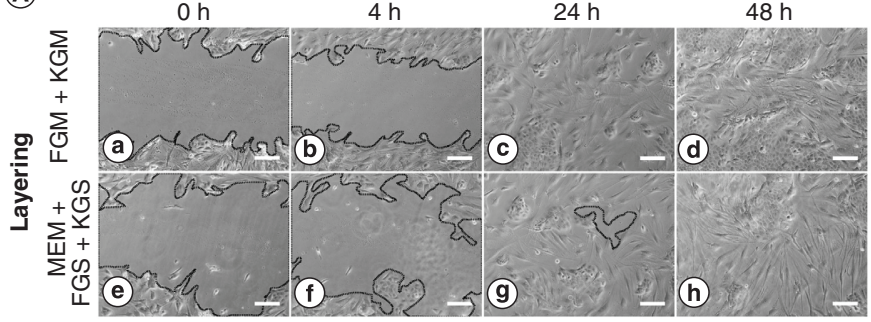

(C) $0 \mathrm{~h}$

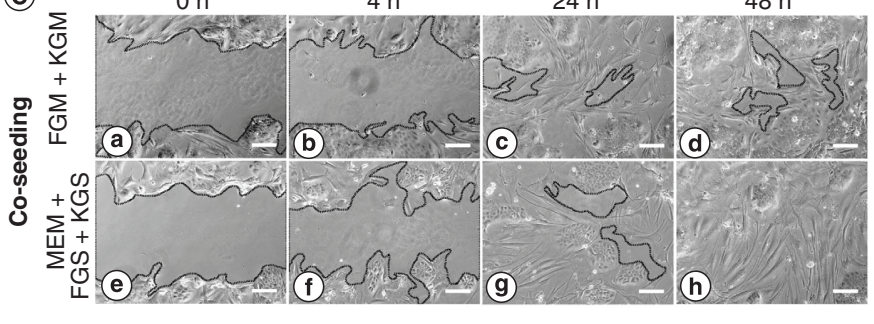

(B)

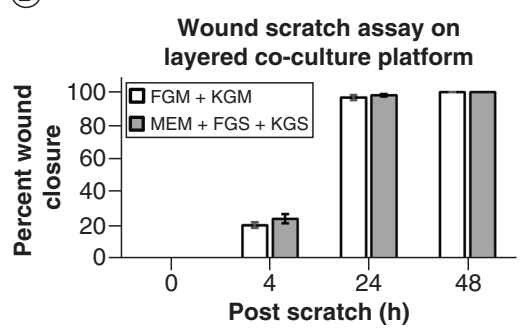

(D)

Wound scratch assay on co-seeded co-culture platform

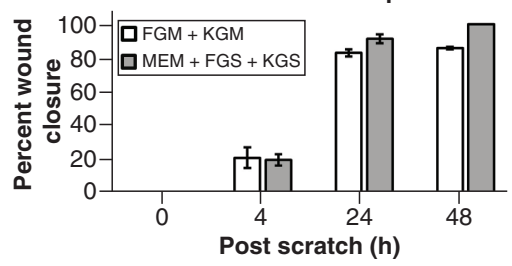

Figure 3. Wound scratch assay for cell migration and wound closure. Co-cultured HDFa and HaCaT cells under reduced serum conditions (layering and co-seeding approaches) were scratched to mimic injury and wound closure was monitored. (A \& B) In the layering approach, HDFa and HaCaT cells start migrating into the wound gap by $4 \mathrm{~h}$, with near closure of the wound gap by $24 \mathrm{~h}$. (C \& D) In the co-seeding approach, HDFa and HaCaT cells start migrating into the wound gap by $4 \mathrm{~h}$, and progressively fill the wound area with $80-100 \%$ closure at $48 \mathrm{~h}$. Error bars represent the standard error of the mean; at least three replicates for each condition.

Scale bars $=150 \mu \mathrm{m}$.

FGM: Fibroblast growth medium; FGS: Fibroblast growth supplement; HDFa: Human dermal fibroblast; KGM: Keratinocyte serum-free growth medium; KGS: Keratinocyte growth supplement; MEM: Minimal essential medium.

absence of serum and growth factor supplements, HDFa cells showed poor proliferation even after 5 days, and HaCaT cells failed to attach and proliferate; in the presence of $10 \%$ FBS alone (no growth supplements), HaCaT showed minimal proliferation, and HDFa failed to form typical confluent sheaths around HaCaT colonies (day 3 onwards; compare with Figure 2). This underscores the need for both optimum serum concentrations and cell-specific growth supplements for successful coculture of HDFa and HaCaT; both of these factors are included in the reduced serum formulations in this study. Previous serum-free approaches have added a range of growth supplements individually, which is both resource- and time-intensive [34,35].

\section{Co-cultured platforms, under reduced serum conditions, enable the study of cell migration \& wound closure using scratch assays}

To enable the study of wound bed functions using the scratch assay, HDFa and HaCaT cells were cocultured in tissue culture-treated six-well plates under reduced serum conditions, FGM+KGM ( $1 \%$ serum) and in MEM+FGS+KGS ( $2 \%$ serum). As seen in Supplementary Figure 2A, using both layering and co-seeding approaches, HDFa and HaCaT cells showed characteristic coculture morphology in tissue culture-treated six-well plates, similar to the results in tissue culture-treated T25 flasks (Figure 2). After the scratch was made, the wound area was imaged at 0, 4, 24 and $48 \mathrm{~h}$. From images for each time point, the wound area was measured (using Image J) and the percentage wound closure was calculated (Supplementary Figure 2B).

In the layering approach under both reduced serum conditions, HDFa and HaCaT cells started filling the wound gap by $4 \mathrm{~h}$, with near-complete closure of the gap by $24 \mathrm{~h}$ (Figure $3 \mathrm{~A} \& \mathrm{~B}$ ). In the co-seeding approach, under both reduced serum conditions, HDFa and $\mathrm{HaCaT}$ proliferation and migration started filling the wound gap by $4 \mathrm{~h}$. The gap continued to be filled between 24 and $48 \mathrm{~h}$, with the wound area showing complete closure (100\%) with MEM+FGS+KGS and $80 \%$ closure for FGM+KGM after $48 \mathrm{~h}$ (Figure $3 \mathrm{C} \& \mathrm{D}$ ). In the first $24 \mathrm{~h}$, the $\sim 20 \%$ closure of the wound gap is likely due to a combination of proliferation and early migration in the periphery of the scratch (wound). Subsequently, the gap was seen to be filled predominantly by migrating HDFa cells, while HaCaT clusters were seen to proliferate (seen as increasing size of HaCaT clusters) and fill the wound area from the periphery.

\section{Conclusion}

The reduced serum approaches in this study can be adopted for coculture of these two cell types for wound and skin studies, tissue bioengineering and cell therapy applications. Until serum alternatives (human plasma, platelet lysate) or synthetic (serum-free) cell culture media are in routine use for fibroblast and keratinocyte culture, and are optimized to support coculture of these cell types, these 
reduced serum approaches will serve to reduce the scientific, technical and ethical limitations associated with the use of animal serum in wound and skin studies [41]. For example, serum-containing media contain high levels of calcium [41]. Although calcium supports cell attachment, signaling, movement and architecture in cell culture studies, in the context of keratinocytes it also facilitates differentiation and cornification [42]. However, in studies to recapitulate the structure and functions of the wound bed (as opposed to skin), media containing high calcium levels is not physiologically representative, nor is further cornification of HaCaT cells relevant to the experiments. Therefore in employing reduced serum concentrations, our approaches provide an additional advantage of lowered calcium levels and a more physiological cocultured wound bed milieu.

\section{Future perspective}

The proposed approaches will also lend well to further modifications and applications. While HaCaT cells serve as a good model platform for keratinocyte studies [30,32,33], a viable next step would be to optimize the coculture of HDFa with primary human epidermal keratinocytes (HEKa). Given the low serum tolerance of $\mathrm{HEKa}$, this could require further reducing the concentration of FBS in the formulations, while retaining that of the growth supplements, to achieve a 'sweet spot' between HDFa serum requirements and HEKa serum tolerance. Another important application of the reduced serum approaches is related to coculturing the recapitulated wound bed with microbes (bacteria, fungi) to mimic an infected wound state. Given that serum is known to be microbicidal $[43,44]$, the presence of reduced concentrations of serum will be advantageous toward optimizing host cell-microbe culture. Finally, while we have developed our approaches in standard cell culture flask and six-well systems, our approaches can be easily taken to 3D coculture and organoid systems, with the addition of matrix and other relevant components.

\section{Executive summary}

- Reduced serum media formulations were developed using commercially available, easy to use cell culture media.

- The formulations support growth of human dermal fibroblasts and immortalized epidermal keratinocytes in both layering and co-seeding approaches in tissue culture-treated flasks and well plates.

- The formulations also allow the study of wound bed functions such as cell migration and wound closure using scratch assays.

\section{Protocols}

- Layering approach of HDFa and HaCaT under reduced serum conditions: dx.doi.org/10.17504/protocols.io.bijukcnw

- Co-seeding approach of HDFa and HaCaT under reduced serum conditions: dx.doi.org/10.17504/protocols.io.bimfkc3n

- Scratch assays with coculture platforms under reduced serum conditions: dx.doi.org/10.17504/protocols.io.bimgkc3w

\section{Supplementary data}

To view the supplementary data that accompany this paper please visit the journal website at: www.future-science.com/doi/suppl/10. 2144/btn-2020-0112

\section{Author contributions}

This study was conceptualized by S Kadam, M Vandana and K Kaushik. The experiments and data analysis were carried out by S Kadam and $\mathrm{M}$ Vandana. The manuscript was written by $\mathrm{S}$ Kadam, $\mathrm{M}$ Vandana and $\mathrm{K}$ Kaushik. The funding for this work was acquired by $\mathrm{K}$ Kaushik. All authors have read and agreed to the published version of the manuscript.

\section{Acknowledgments}

We thank M Motwani and S Asai, Linq Labs, Jehangir Clinical Development Centre, Pune, India for the HaCaT cell line. We thank A Teli, S Gayan and J Dave (Institute of Bioinformatics and Biotechnology, Savitribai Phule Pune University) for technical inputs on microscopy.

\section{Financial \& competing interests disclosure}

This study was funded by the Ramalingaswami Re-entry Fellowship and Har Gobind Khorana-Innovative Young Biotechnologist Award, Department of Biotechnology, Government of India (to K Kaushik, BT/RLF/Re-entry/11/2015 and BT/12/IYBA/2019/05). The authors have no other relevant affiliations or financial involvement with any organization or entity with a financial interest in or financial conflict with the subject matter or materials discussed in the manuscript apart from those disclosed.

No writing assistance was utilized in the production of this manuscript.

\section{Open access}

This work is licensed under the Attribution-NonCommercial-NoDerivatives 4.0 Unported License. To view a copy of this license, visit http://creativecommons.org/licenses/by-nc-nd/4.0/ 


\section{References}

Papers of special note have been highlighted as: $\bullet$ of interest; $\bullet \bullet$ of considerable interest

1. Gurtner GC, Werner S, Barrandon Y, Longaker MT. Wound repair and regeneration. Nature 453(7193), 314-321 (2008).

2. Landén NX, Li D, Ståhle M. Transition from inflammation to proliferation: a critical step during wound healing. Cell. Mol. Life Sci. 73(20), 3861-3885 (2016).

3. Li J, Kirsner RS. Wound Healing. In: Surgery of the Skin. Elsevier Inc. 97-115 (2005).

4. Sorrell JM, Caplan Al. Fibroblast heterogeneity: more than skin deep. J. Cell Sci. 667-675 (2004).

5. Wojtowicz AM, Oliveira S, Carlson MW, Zawadzka A, Rousseau CF, Baksh D. The importance of both fibroblasts and keratinocytes in a bilayered living cellular construct used in wound healing. Wound Repair Regen. 22(2), 246-255 (2014).

- Highlights the importance and functions of fibroblasts and keratinocytes in the wound bed and in wound healing.

6. Menon SN, Flegg JA, Mccue SW, Schugart RC, Dawson RA, McElwain SDL. Modelling the interaction of keratinocytes and fibroblasts during normal and abnormal wound healing processes. Proc. Biol. Sci. 279(1741), 3329-3338 (2012).

7. Ghahary A, Ghaffari A. Role of keratinocyte-fibroblast cross-talk in development of hypertrophic scar. Wound Repair Regen. S46-S53 (2007).

8. Werner S, Krieg T, Smola H. Keratinocyte-fibroblast interactions in wound healing. J. Invest. Dermatol. 127(5), 998-1008 (2007).

9. Sriram G, Bigliardi PL, Bigliardi-Qi M. Fibroblast heterogeneity and its implications for engineering organotypic skin models in vitro. Eur. J. Cell Biol. 483-512 (2015).

10. Wang Z, Wang Y, Farhangfar F, Zimmer M, Zhang Y. Enhanced keratinocyte proliferation and migration in coculture with fibroblasts. PLoS ONE 7(7), e40951 (2012).

11. Walter MNM, Wright KT, Fuller HR, MacNeil S, Johnson WEB. Mesenchymal stem cell-conditioned medium accelerates skin wound healing: an in vitro study of fibroblast and keratinocyte scratch assays. Exp. Cell Res. 316(7), 1271-1281 (2010).

-. Uses fibroblast and keratinocye coculture platforms to study patterns of wound bed cell migration upon injury with scratch assays.

12. Gabbott CM, Sun T. Comparison of human dermal fibroblasts and hacat cells cultured in medium with or without serum via a generic tissue engineering research platform. Int. J. Mol. Sci. 19(2), 388 (2018).

•• Coculture of human dermal fibroblasts (HDFa) and immortalized human epidermal keratinocytes (HaCaT) in the presence of serum (10\% FBS) and under serum-free conditions. Both approaches were suboptimal for coculturing $\mathrm{HDFa}$ and $\mathrm{HaCaT}$ cells.

13. Maas-Szabowski N, Stärker A, Fusenig NE. Epidermal tissue regeneration and stromal interaction in HaCaT cells is initiated by TGF- $\alpha$. J. Cell Sci.116(Pt 14), 2937-2948 (2003).

14. Jubin K, Martin Y, Lawrence-Watt DJ, Sharpe JR. A fully autologous coculture system utilising non-irradiated autologous fibroblasts to support the expansion of human keratinocytes for clinical use. Cytotechnology 63(6), 655-662 (2011).

15. Sato T, Kirimura Y, Mori Y. The coculture of dermal fibroblasts with human epidermal keratinocytes induces increased prostaglandin E2 production and cyclooxygenase 2 activity in fibroblasts. J. Invest. Dermatol. 109(3), 334-339 (1997).

16. Wang TW, Huang YC, Sun JS, Lin FH. Keratinocyte-fibroblast cocultures on a bi-layered gelatin scaffold for skin equivalent tissue engineering. J. Med. Biol. Eng. 23(4), 213-220 (2003).

17. Sun T, McMinn P, Holcombe M, Smallwood R, MacNeil S. Agent based modelling helps in understanding the rules by which fibroblasts support keratinocyte colony formation. PLoS ONE 3(5), e2129 (2008).

- Indicates the importance of contact-based coculture of fibroblasts and keratinocytes (as opposed to transwell systems) to mimic wound bed structure/functions and skin functions.

18. Klicks J, von Molitor E, Ertongur-Fauth T, Rudolf R, Hafner M. In vitro skin three-dimensional models and their applications. J. Cell. Biotechnol. 3(1), 21-39 (2017).

19. Tan J, Zhao C, Zhou J et al. Co-culturing epidermal keratinocytes and dermal fibroblasts on nano-structured titanium surfaces. Mater. Sci. Eng. C. 78 , 288-295 (2017).

20. Shipley GD, Keeble WW, Hendrickson JE, Coffey RJ, Pittelkow MR. Growth of normal human keratinocytes and fibroblasts in serum-free medium is stimulated by acidic and basic fibroblast growth factor. J. Cell. Physiol. 138(3), 511-518 (1989).

21. Seeger MA, Paller AS. The roles of growth factors in keratinocyte migration. Adv. Wound Care 4(4), 213-224 (2015).

22. Peplow PV, Chatterjee MP. A review of the influence of growth factors and cytokines in in vitro human keratinocyte migration. Cytokine 62(1), 1-21 (2013).

23. Yun YR, Won JE, Jeon E et al. Fibroblast growth factors: biology, function, and application for tissue regeneration. J. Tissue Eng. 2010, 218142 (2010).

24. Sun T, Higham M, Layton C, Haycock J, Short R, MacNeil S. Developments in xenobiotic-free culture of human keratinocytes for clinical use. Wound Repair Regen. 12(6), 626-634 (2004).

25. Lamb R, Ambler CA. Keratinocytes propagated in serum-free, feeder-free culture conditions fail to form stratified epidermis in a reconstituted skin model. PLoS ONE 8(1), e52494 (2013).

26. Richards S, Leavesley D, Topping G, Upton Z. Development of defined media for the serum-free expansion of primary keratinocytes and human embryonic stem cells. Tissue Eng. Part $C$ Methods 14(3), 221-232 (2008).

27. Jackson C, Aabel P, Eidet JR et al. Effect of storage temperature on cultured epidermal cell sheets stored in xenobiotic-free medium. PLoS ONE 9(8), e105808 (2014).

28. Jayme DW, Daley JP, Price PJ. Serum-free media for the growth and maintenance of the differentiated state of keratinocytes, hepatocytes, and neural cells. In: Animal Cell Technology: Basic \& Applied Aspects. Springer, Dordrecht, The Netherlands, 327-334 (1992).

29. Schurer N, Kohne A, Schliep V, Barlag K, Goerz G. Lipid composition and synthesis of HaCaT cells, an immortalized human keratinocyte line, in comparison with normal human adult keratinocytes. Exp. Dermatol. 2(4), 179-185 (1993).

30. Deyrieux AF, Wilson VG. In vitro culture conditions to study keratinocyte differentiation using the HaCaT cell line. Cytotechnology 54(2), 77-83 (2007).

31. Boukamp P, Petrussevska RT, Breitkreutz D, Hornung J, Markham A, Fusenig NE. Normal keratinization in a spontaneously immortalized aneuploid human keratinocyte cell line. J. Cell Biol. 106(3), 761-771 (1988).

32. Micallef L, Belaubre F, Pinon A et al. Effects of extracellular calcium on the growth-differentiation switch in immortalized keratinocyte HaCaT cells compared with normal human keratinocytes. Exp. Dermatol. 18(2), 143-151 (2009).

33. Colombo I, Sangiovanni E, Maggio R et al. HaCaT cells as a reliable in vitro differentiation model to dissect the inflammatory/repair response of human keratinocytes. Mediators Inflamm. 2017, 7435621 (2017).

-. Substantiates that HaCaT cells (immortalized human keratinocytes) serve as a reliable model system for keratinocyte functions.

34. Higham MC, Dawson R, Szabo M, Short R, Haddow DB, Macneil S. Development of a stable chemically defined surface for the culture of human keratinocytes under serum-free conditions for clinical use. Tissue Eng. 9(5), 919-930 (2003).

35. Auxenfans C, Thépot A, Justin V et al. Characterisation of human fibroblasts as keratinocyte feeder layer using p63 isoforms status. Biomed. Mater. Eng. 19(4-5), 365-372 (2009).

36. Llames S, García-Pérez E, Meana Á, Larcher F, Del Río M. Feeder layer cell actions and applications. Tissue Eng. Part B Rev. 21(4), 345-353 (2015).

37. Bisson F, Rochefort É, Lavoie A et al. Irradiated human dermal fibroblasts are as efficient as mouse fibroblasts as a feeder layer to improve human epidermal cell culture lifespan. Int. $J$. Mol. Sci. 14(3), 4684-4704 (2013).

38. Kamano Y, Egusa H, Okawa H, Yatani H. Feeder cell sources and feeder-free methods for human ips cell culture. In: Interface Oral Health Science 2014: Innovative Research on Biosis-Abiosis Intelligent Interface. Springer, Japan, 145-159 (2015).

39. Abràmoff MD, Magalhães PJ, Ram SJ. Image processing with imageJ. Biophotonics Int. 36-42 (2004)

40. Mujaj S, Manton K, Upton Z, Richards S. Serum-free primary human fibroblast and keratinocyte coculture. Tissue Eng. Part A. 16(4), 1407-1420 (2010).

41. Yao T, Asayama Y. Animal-cell culture media: history, characteristics, and current issues. Reprod. Med. Biol. 16(2), 99-117 (2017).

42. Bikle DD, Xie Z, Tu CL. Calcium regulation of keratinocyte differentiation. Expert Rev. Endocrinol. Metab. 7(4), 461-472 (2012).

43. Berciu I, Săndulescu O, Streinu-Cercel A, Streinu-Cercel A. Serum inhibitory and bactericidal titers in the clinical management of bacterial infections. BMC Infect. Dis. 14(S7), 1-2 (2014).

44. Necchi F, Saul A, Rondini S. Development of a high-throughput method to evaluate serum bactericidal activity using bacterial ATP measurement as survival readout. PLoS ONE 12(2), e0172163 (2017). 
